\title{
Use and Users of the Web-Based Omaolo Covid-19 Symptom Self-Assesment Tool in Finland Since March 16, 2020
}

\author{
Vesa JORMANAINEN ${ }^{\mathrm{a}, \mathrm{b}, 1}$ and Leena SOININEN ${ }^{\mathrm{c}}$ \\ ${ }^{\mathrm{a}}$ Finnish Institute for Health and Welfare (THL), Helsinki, Finland \\ ${ }^{b}$ University of Helsinki, Helsinki, Finland \\ ${ }^{\mathrm{c}}$ DigiFinland $\mathrm{Oy}$, Helsinki, Finland
}

\begin{abstract}
In Finland, it is possible to quickly produce medical symptom selfassessment tools within the existing infrastructure. The Finnish Omaolo Covid-19 web-based symptom self-assessment tool (symptom checker) was launched on March 16, 2020 after a 6-day development period. By using the web-based Omaolo Covid-19 symptom checker during the second wave of the epidemic, some 1.72 million questionnaires were recorded, out of which 1.55 million from symptomatic persons. Some $15 \%$ of the responses $(245,500)$ were directed to seek emergency medical care based on the online screening by respondent response profiles.
\end{abstract}

Keywords. Covid-19, symptom assessment, digital epidemiology, Finland

\section{Introduction}

Rapid pace of the Covid-19 pandemic presents challenges to robust collection of population-scale data to address the pandemic. Symptom information is vital to providing an earlier window into our progress against viral spread, and can complement traditional measures of the pandemic's severity [1-4].

Mobile apps provide a convenient source of tracking and data collection to fight against the spread of Covid-19 [5-7]. Digital surveillance as a method of contactless tracing holds substantial appeal for the future [2,8-10]. However, mobile phone apps and web-based tools facilitate self-guided collection of population-level data at scale [5,11-14], even in real-time [15] but with varying efficacy [16].

Symptom checkers are algorithm-based programs that provide potential virtual diagnoses and triage advice [17-19]. Symptom checkers promise a cost-effective way of enabling rapidly scalable epidemiological data collection and analysis, with varying diagnostic accuracy [20,21].

Covid-19 epidemic in Finland started in Mid-March 2020. On March 16, 2020 the Finnish Government announced a state of emergency due to the Covid-19 epidemic and consequently implemented several physical distancing measures aimed at slowing

\footnotetext{
${ }^{1}$ Corresponding Author, Vesa Jormanainen, Performance Assessment of the Health and Social Service System, National Institute for Health and Welfare (THL), P.O. Box 30, 00271 Helsinki, Finland; E-mail: vesa.jormanainen@thl.fi.
} 
the spread and protecting risk groups [22]. Part of the national response was the Omaolo Covid-19 web-based symptom self-assessment tool, a CE-marked medical device that was launched March 16, 2020 [23].

Our objective is to describe use, users, and some performance aspects of the Finnish Omaolo Covid-19 web-based symptom self-assessment tool (symptom checker) in Finland.

\section{Material and Method}

Development of the web-based Omaolo Covid-19 symptom checker was started March 10, 2020, and it was launched for use March 16, 2020. It has been published in Finnish and Swedish (March 2020) and in English (April 2020) versions. All symptom checker responses that were started are recorded into the DigiFinland Oy's system log files. However, the respondent decides if one would record the questionnaire responses to the system containing personal identification codes. By recording the responses, one has to authenticate oneself by strong measures, and can transmit one's profile responses further to one's home municipality primary healthcare centre processing by given consent.

Our study material consists of daily Omaolo Covid-19 symptom checker responses since March 16, 2020. Data are statistical and do not contain any variables or their combinations that could be used to identify any person in the material.

We defined Covid-19 epidemic in two waves based on its epidemiological features in Finland: the first wave from February 25 to June 28, 2020, and the second wave from June 29, 2020 to January 30, 2021.

\section{Results}

In total, there were 1,937,469 responses recorded into the Omaolo Covid-19 web-based symptom checker system log from March 16 to December 31, 2020, out of which 1,536,625 (79\%) during the second wave of the Covid-19 epidemic in Finland (Figure 1).

Most $(635,757$ of $1,517,164$; e.g. $42 \%)$ of the responses were recorded in the Helsinki and Uusimaa University Hospital District. Based on questionnaire incidence per 100,000 inhabitants, the three top ones were Helsinki and Uusimaa (45,094), PäijätHäme $(33,948)$, and Keski-Suomi $(31,841)$ Hospital Districts whereas the three lowest incidences were in Länsi-Pohja (11,226), Lapland (12,719), and Eastern Finland $(14,596)$ Hospital Districts, respectively.

Only symptomatic persons answered questions regarding symptoms (Figure 2). The questionnaire symptoms were: cough, difficulty in breathing, throat ache, headache, muscle pain, vomiting or diarrhea, loss of smell or taste sense, and fever $(>37.5 \mathrm{C})$. Fever occurred in mean in $12 \%$ of the responses. The proportions of symptoms occurred in relatively constantly during the second wave, throat ache, cough and fever being the most prevalent symptoms. Vomiting or diarrhea occurred in $2.6 \%$ and loss of smell or taste sense in less than $1 \%$ of the responses. 


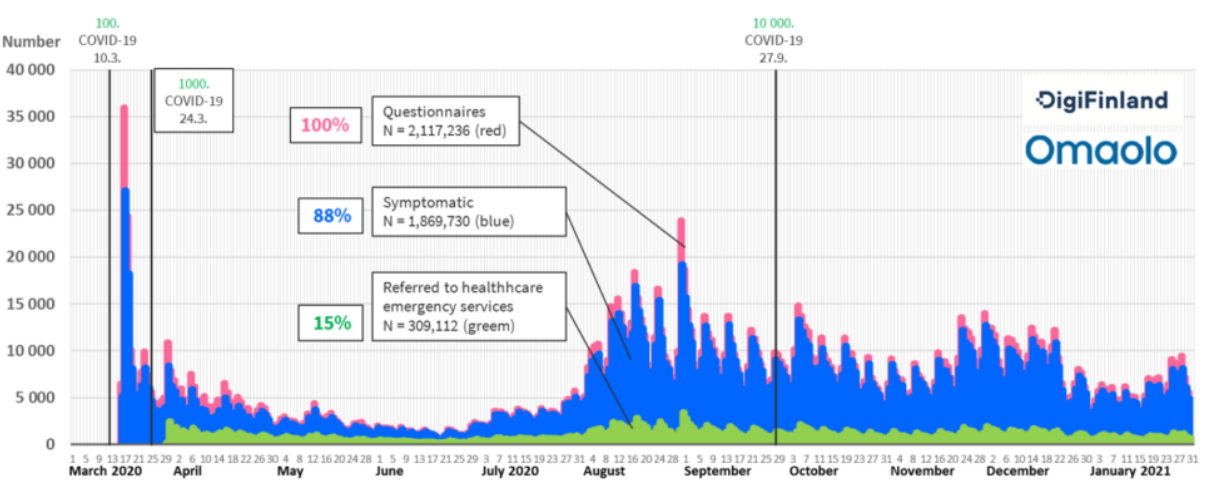

Figure 1. Daily number of web-based Omaolo Covid-19 symptoms self-assessment questionnaires, symptomatic and cases referred to seek healthcare emergency service from March 16, 2020 to January 30, 2021 in Finland. In a total of 2,117,236 responses 1,937,469 were from March 16 to December 31, 2020 and 1,716,392 responses from June 29, 2020 to January 30, 2021 (during the second wave).

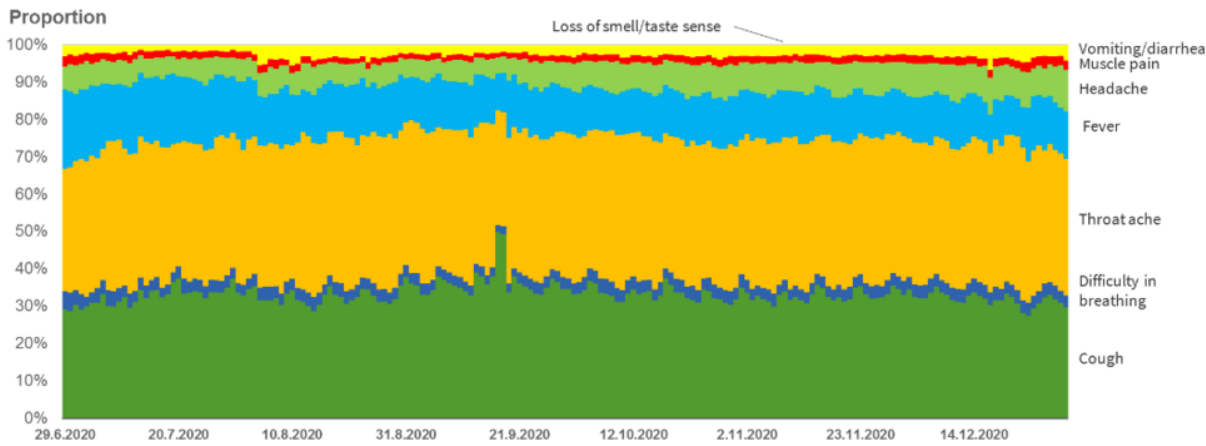

Figure 2. Daily number of symptoms in web-based Omaolo Covid-19 symptoms self-assessment questionnaires during the second wave of the epidemic from June 29, 2020 to January 30, 2021 in Finland $(\mathrm{n}=1,386,739)$. One respondent may have reported many symptoms. All symptoms for one day were counted together $(100 \%)$ as a sum of symptoms for the day. A symptom proportion was calculated by dividing the symptom frequency by the symptom sum for the day, and multiplied by 100 .

Respondents' age was classified into 10-year age groups. Compared with the age distribution in the whole country, the proportions were larger in younger age groups (less than 50-year-olds) in the symptom checker respondents whereas smaller in older age groups. The differences were most marked among the $20-29$-year-olds $(25 \%$ in symptom checker and $12 \%$ among total population), 30-39-year-olds (22\% vs. $13 \%)$, $60-69$-year-olds (6\% vs. $13 \%)$ and 70 -year-old or older ( $2 \%$ vs. $16 \%)$.

In total, $13 \%(176,440)$ of the respondents during the second wave were working in social welfare or healthcare, $30 \%$ in other services where the respondent could not escape from close contacts with customers, $26 \%$ in services where the respondent was able to escape from close contacts, and $31 \%$ were not working at the time of response. 
The respondent were standing and could perform their normal domestic duties in $87 \%$ of responses, whereas $13 \%$ were standing but could not perform their normal domestic duties, and $0.8 \%$ were in weak condition and could only stay in bed (excluding bathroom visits).

There were 514,143 (33\%) respondents who claimed being (directly) exposed to Covid-19 during the second wave of the epidemic. The proportion varied between 40 $45 \%$ from March to August, 2020 but decreased to 25-30\% towards the end of 2020.

Respondents were asked to identify if they had diseases, treatments or states that may lower their response to infections. Self-reported risk group identification occurred in $10-12 \%$ from March to mid-August whereas $8-10 \%$ thereafter.

Based on the responses given in the web-based symptom checker questionnaire, the respondent was given an evaluation of the probability of Covid-19 disease, advice how to prevent spreading the Covid-19 disease, and how to seek medical help in acute cases. The recommendations were classified based on their priority into three major groups: no need for treatment, low or high priority for treatment (17 subclasses in total). There were 220,535 group 2 (high priority) responses, i.e. 14\% of all responses during the second wave of the epidemic. The proportion was higher (some 20\%) during the June-July and the trend decreased to $10-15 \%$ thereafter.

\section{Discussion and Conclusions}

By using the web-based Omaolo Covid-19 symptom checker during the second wave of the epidemic, some 1.72 million questionnaires were recorded, out of which 1.55 million from symptomatic persons. Some $15 \%$ of the responses $(245,500)$ were directed to seek emergency medical care based on the online screening. The case load avoided at emergency services by utilizing digital web-based tool is most probably a convenient way to conduct digital surveillance as a method of contactless tracing, to facilitate self-guided collection of population-level data at scale in real-time.

The symptom checker users were predominantly younger than the general population in Finland, which may be explained by easier access, better skills and conventional habits in using digital apps. Most responses were recorded in the capital Helsinki area corresponding to the epidemiological situation. Some $13 \%$ were working in social welfare and healthcare, and one out of ten was in a risk group by selfassessment. It is possible that those who proceed with milder symptom profiles may be provided with a possibility to enter primary healthcare provision in excess to others via the Omaolo Covid-19 web-based symptom checker tool. This is also the case for entering to testing basing on the symptom checker tool profiles.

In Finland, it is possible to quickly produce medical symptom assessment tools for symptom checker use with treatment guidance for various use cases within the existing infrastructure. This study presents and describes use, users, and some performance aspects of the Finnish Omaolo Covid-19 web-based symptom self-assessment tool (symptom checker) since its launch on March 16, 2020 and during the second wave of the Covid-19 epidemic in Finland since June 29, 2020. The CE-marked medical device was launched after a 6-day development period.

Unfortunately in this study we were not able to follow how the respondent made actions, such as going to a Covid-19 test. However, the Omaolo symptom checker is accepted by the population and is used frequently to self-assess Covid-19 symptoms. Its use frequencies have been observed to correlate with Covid-19 confirmed cases. 


\section{References}

[1] Chan AT, Brownstein JS. Putting the public back in public health: surveying symptoms of Covid-19. NEJM 2020;383:e45(1-3).

[2] Budd J, Miller BS, Manning EM, Lampos V, Zhung M, Edelstein M, et al. Digital technologies in the public-health response to CIVID-19. Nature Med. 2020;20:1183-1192.

[3] Alimohamedi Y, Sepandi M, Taghidir T, Homsamirudsari H. Determine the most common clinical symptoms in COVID-19 patients: a systematic review and meta-analysis. J. Prev. Med. Hyg. 2020;61:E304-E312.

[4] Struyf T, Deeks JJ, Dinnes J, Takwoingi Y, Davenport C, Leefland MMG, et al. Signs and symptoms to determine if a patient presenting in primary care or hospital outpatient setting has COVID-19 disease. Cochrane Database of Systematic Reviews. 2020(7):CD013665.

[5] Alwashmi MF. The use of digital health in the detection and management of COVID-19. Int. J. Environ. Res. Public Health 2020;17:2906.

[6] Drew DA, Nguyen LH, Steves CJ, Menni C, Freydin M, Varsavsky T, et al. Rapid implementation of mobile technology for real-time epidemiology of COVID-19. Science 2020;368:1362-1367.

[7] Sharma T, Bashir M. Use of apps in the COVID-19 response and the loss of privacy protection. Nature Med. 2020;26:1160-1169.

[8] Whitelaw S, Mamas MA, Topol E, Van Spall HCG. Applications of digital technology in COVID-19 pandemic planning and response. Lancet Digital Health 2020;2:e435-e440.

[9] Ferretti L, Wyman C, Kendall M, Zhao L, Nurtay A, Abele-Dörner L, et al. Quantifying SARS-CoV-2 transmission suggests epidemic control with digital contact tracing. Science 2020;368:619.

[10] Trang S, Trenz M, Weiger WH, Tarafdar M, Cheung CMK. One app to trace them all? Examining app specifications for mass acceptance of contact-tracing apps. Eur. J. Inform. Syst. 2020.

[11] Judson TJ, Odisho AY, Neinstein AB, Chao J, Williams A, Miller C, et al. Rapid design and implementation of an integrated patient self-triage and self-scheduling tool for COVID-19. J. Am. Med. Inform. Assoc. 2020;27:860-866.

[12] Miner AS, Larajo L, Kocoballi AB. Chatbots in the fight against the COVID-19 pandemic. npj Digital Med. 2020;3:65.

[13] Rossman H, Keshet A, Shilo S, Gavrieli A, Bauman T, Cohen O, et al. A framework for identifying regional outbreak and spread of COVID-19 from one-minute population-wide surveys. Nature Med. 2020;26:632-638.

[14] Ye Q, Zhou J, Wu H. Using information technology to manage the COVID-19 pandemic: development of a technical framework based on practical experience in China. JMIR Med. Inform. 2020;8:e19515.

[15] Menni C, Valdes AM, Freidin MB, Sidre CH, Nguyen LH, Drew DA, et al. Real-time tracking of selfreported symptoms to predict potential COVID-19. Nature Med. 2020;26:1037-1040.

[16] Callahan A, Steinberg E, Fries JA, Gombar S, Patel B, Corbin CK, et al. Estimating the efficacy of symptom-based screening for COVID-19. npj Digital Med. 2020;3:95.

[17] Gasser U, Ienca M, Scheibner J, Slegh J, Vayena E. Digital tools against COVID-19: taxonomy, ethical challenges, and navigations aid. Lancet Digital Health. 2020;2:e425-e434.

[18] Hill MG, Sim M, Mills B. The quality of diagnosis and triage advice provided by free online symptom checkers and apps in Australia. MJA. 2020;212:514-519.

[19] Meyer AND, Giardina TD, Spitzmueller C, Shadid U, Scott TMT, Singh H. Patient perspectives on the usefulness of an artificial intelligence-assisted symptom checker: cross-sectional survey study. J. Med. Internet Res. 2020;22:e14679.

[20] Mansab F, Bhatti S, Goyal D. The performance of national COVID-19 'symptom checkers': a comparative case simulation study. medRxiv. Nov 30, 2020.

[21] Munsch N, Martin A, Gruarin S, Natoqi J, Abdarahmane I, Weingartner-Ortner R, et al. Diagnostic accuracy of web-based COVID-19 symptom checkers: comparison study. J. Med. Internet Res. 2020;22:e21299.

[22] Tiirinki H, Tynkkynen L-K, Sovala M, Atkins S, Koivusalo M, Rautiainen P, et al. COVID-19 pandemic in Finland: preliminary analysis on health system response and economic consequences. Health Policy Technol. 2020;9:649-662.

[23] Jormanainen V, Kaikkonen R, Isosomppi S, Numminen J, Kunnamo I, Salaspuro T, et al. The Finns quickly found the coronavirus symptom assessment online service: those who responded to the Omaolo coronavirus symptom assessment from 16 March to 15 May, 2020. Discussion Paper 27/2020. Helsinki: Finnish Institute for Health and Welfare (THL). 30 pages. [In Finnish, Abstract in English]. 